\title{
Semantic Annotation of Resources of Distance Learning based Intelligent Agents
}

\author{
http://dx.doi.org/10.3991/ijep.v4i1.2845 \\ Chekry Abderrahman, Oriche Aziz and Khaldi Mohamed \\ Abdelmalek Essaâdi University, Lirosa, Tetwan, Morocco
}

\begin{abstract}
This paper presents a system based on intelligent agents for the semantic annotation of learning resources taking into account the context of training. Semantic annotations systems rarely treat existing semantic annotations in the field of distance education (e-learning). Most researchers in the field of education limit annotations to specific cases (teacher annotation, learner annotation, annotation of electronic documents etc.) these annotations are edited by users with annotation tools. In turn, in our approach, we propose a semantic annotation system based on intelligent agents that manage semantic annotations of educational resources; these annotations are guided by domain of ontologies and ontology applications. We believe that the original resource annotations, a storehouse of learning objects, are standardized by LOM profile, these learning objects are managed using an ontology learning.
\end{abstract}

Index Terms-Semantic annotation of educational resources; Semantic Web; Metadata; Multiagent Systems; Ontologies.

\section{INTRODUCTION}

Many documents, and generally many resources, can be used as part of an e-Learning training. Some of these resources are produced "in house" by the various actors involved in training, others are available on the web: online courses, course materials, supporting oral presentations (slides), bibliographies, frequently asked questions, lecture notes, etc.

To meet the ever-increasing number of resources, the search engines should be able to provide more detailed answers and handle more complex queries integrating knowledge of the user. Given the need for a formal description of the content of educational materials online, we see that it is interesting to integrate software agents able to label semantically textual and multimedia resources, metadata, on the one hand, and to exploit and to improve information retrieval, on the other hand. The formalization of existing web pages is defined as the task of semantic annotation and automatic.

The existing automatic annotation systems are essentially syntactic. Automatic annotation of semantic aspects of web pages requires solving the problem of identifying the appropriate concepts to the content of each web resource and the profile of each user type, vis-à-vis ontologies

Current data from the web are too often written in natural language, as for humans. Natural language is inherently ambiguous too, formal and explicit semantic alternatives should be implemented to eliminate the ambiguities of natural language, both in the content of resources and their annotations. The task of annotation for the semantic web is therefore to take as input a resource document and as outputting the same enriched content with semantic annotations based on representations of knowledge more or less formal [5] content.

Hence, this paper aims to describe the content of educational resources by annotating them with unambiguous information to facilitate the exploitation of these resources by software agents to manage and annotate educational content available to learners and teachers in training online.

\section{ORGANiZATION OF E-LEARNING TRAINING}

\section{A. Management systems of educational resources}

Management systems of educational resources allow, on the one hand, to share resources, information, knowledge through automatic learning, and on the other hand, to better index and manage relationships between them. Knowledge to manage that are of two types (Figure $1)$ :

- Educational concepts to understand;

- Ontological resources describing formal and semantic concepts and relationships between concepts.

These systems use ontologies to share resources, information and knowledge training. The definition of the concepts to be addressed uses ontologies to define a common vocabulary between training actors. The ontology construction work requires a clarification of the concepts to teach and the relationships they maintain and help to clarify the actual content of training.

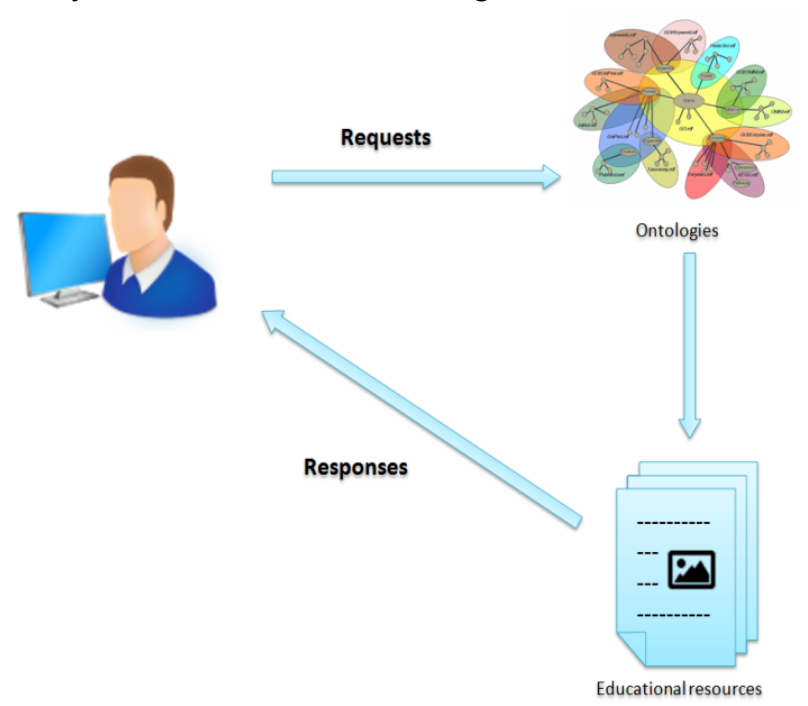

Figure 1. General diagram of a system of management of educational resources 
The implementation of such an approach entails the development of a methodological approach that integrates dimensions from different areas of research:

- Educational engineering for determining metadata concepts to address (concepts) and relations between these concepts;

- Knowledge Engineering for content management training using ontologies;

- The Semantic Web for structuring and semantic annotation of learning resources.

Different work types (Management systems of educational resources) were had their focus in knowledge management based on ontologies to annotate the concepts of learning, other projects are based on the metadata. In our approach we propose another way to annotate educational resources based on intelligent agents.

\section{B. Multi-agent systemes}

In online training users are distributed and heterogeneous. Therefore, it is interesting that the system architecture is itself heterogeneous and distributed. Advances in programming have been made through abstractions increasingly high for us to model systems more complex. MAS ${ }^{1}$ are candidates to be the next step in the levels of abstraction, to understand, model and develop distributed systems [9]. In addition, MAS is recognized as software architecture to support the deployment of the Semantic Web [10].

MAS are a loosely coupled network of agents who work together as a society to resolve problems that are usually beyond the capabilities of any individual agent. MAS are heterogeneous agents when it includes at least two types. A MAIS ${ }^{2}$ is an MAS to provide part or a full range of features for managing and exploiting information resources. When applying MAS on training systems online, the cooperation of agents aims to improve capitalization of knowledge in training.

A MAS architecture is a structure that depicts the different families of agents and their relations. A configuration is the instantiation of architecture with a chosen arrangement and an appropriate number of agents of each type. For a given architecture, we can generate multiple configurations, and a given configuration is closely related to the topography and the context of where it is deployed (training structure, characteristics of the intranet, localization of interest, etc...). Thus, the architecture must be designed so that all possible configurations cover various possible organizational contexts.

\section{Adaptive $M A S$}

An adaptive MAS can be seen as the superposition of three components (Figure 2):

- Component of the field agents: Includes agents of the same type having the ability to resolve a part of overall problem. They operate independently and according to the evolution of their environment. All agents are combined in a conventional network of acquaintance, the network defines the explicit communication between agents;

\footnotetext{
${ }^{1}$ MAS: Multi-Agent Systems

${ }^{2}$ MAIS: Multi-Agent Information System
}

- Adaptive component: Represents the organization established in the field of agents, it is defined by a set of nodes connected together form the network of interdependence, each node of the network corresponds to a field agent, and connections between these nodes represent dependencies between these agents;

- Component coupling: Composed of a set of monitors agents whose role is to continuously observe the first component (field agents), and establish a state of the system and provide this information to the adaptive component.

\section{Store of educational objects}

The establishment of a store of educational objects represents a substantial challenge for the success of eLearning project. This implies new requirements for educational objects. One of them - the main we believe - is to allow the documents to be reused in different contexts to meet several purposes [1].

The store of educational objects is composed of two parts:

- The database that will store all learning objects filtered;

- All LOM descriptors and semantic descriptors resulting from the indexing phase.

LOM metadata and semantic descriptors will allow to easily find a learning object stored. So, our warehouse will have the following structure [1] :

\section{CONTRIBUTION OF AdAPTIVE MAS IN E- LEARNING}

\section{A. Semantic Annotation Of the Educational Resource Based Intelligent agents}

In the process of semantic annotation, ontologies play a crucial role since they model the concepts, their attributes and the relationships are used to annotate the content of the documents [5]. Semantic annotations based on ontologies consider only resources listed in these ontologies and do not completely describe the origins of these resources (Figure. 3).

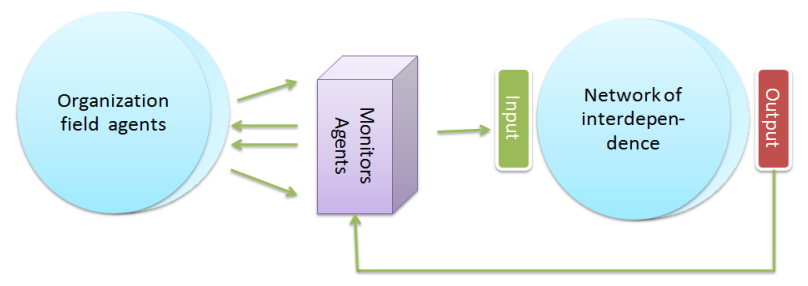

Figure 2. Various components of an adaptive MAS.

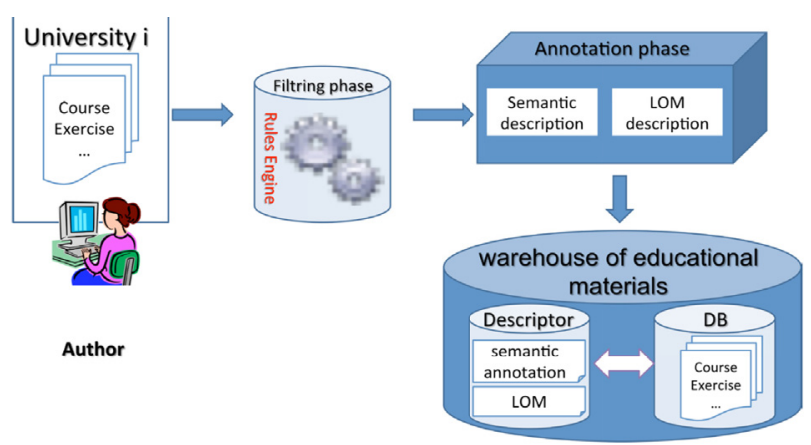

Figure 3. Modeling a warehouse of learning objects. 
One proposed solution to overcome this problem is OntoPop [5] tool solution. This tool presents a solution to semantic annotation and ontology population of source documents based on ANLP ${ }^{3}$ tools. This solution is helpful for annotating general documents. We can not apply to annotate educational objects because of problem of identifying educational concepts. In a press document, for example, we annotate the words of the document, by cons in an educational document the educational concepts contained in this document are annotated, an educational concept can be a word, a sentence or even a chapter.

For this, we see that it is interesting to integrate software agents that are able to label semantically educational resources, metadata, on the one hand, and to improve information retrieval, on the other hand.

Educational resources should be structured to facilitate the generation of semantic annotations of these resources. The reason why we require that each educational resource should be described with LOM metadata is because metadata is represented semantically using ontology learning.

We consider an intelligent agent to manage the semantic annotations of educational objects (Figure. 5); the agent IAMSA $^{4}$ for the generation and validation of annotations.

This agent is in collaboration with $\mathbf{A M O}^{5}$. It confirms updates of educational ontologies in case of modification of one of these concepts by training administrators.

\section{B. The Intelligent Agents in the Heart of Training}

Our IAMSA must be able to semantically annotate the concepts that need to be improved in ontologies training. With the methodologies for information extraction from educational resources, we need ANLP engine to identify the pedagogical concepts to annotate.

The AMO (Figure. 6) deals with the ontological training environment. In case there is an update of ontologies by administrator, AMO sends a message to the IAMSA which contains the type of modification made and the concept will be modified so that the agent will update the annotations corresponding to this concept. In the case of deleting one or more concepts, AMO handles the formal reconstruction of the ontology in question.

The $\mathbf{R A}^{\mathbf{6}}$ allows to find the appropriate response to the user from the set of answers generated by the system.

\section{CONClusion}

We have presented an innovative approach to managing online training by combining ontology engineering, semantic web and multi-agent systems in an integrated solution. The integration of knowledge engineering approach (formalization of knowledge resources dispersed online with semantic annotations based on ontologies) and artificial intelligence (information systems of several coupled cooperation agents at semantic level) can be a new powerful paradigm for the design of an online course.

${ }^{3}$ ANLP: Automatic Natural Language Processing.

${ }^{4}$ IAMSA: Intelligent Agent Manager Semantic Annotations.

${ }^{5}$ AMO : Agent Manager Ontologies.

${ }^{6}$ RA: Relevance Agent.

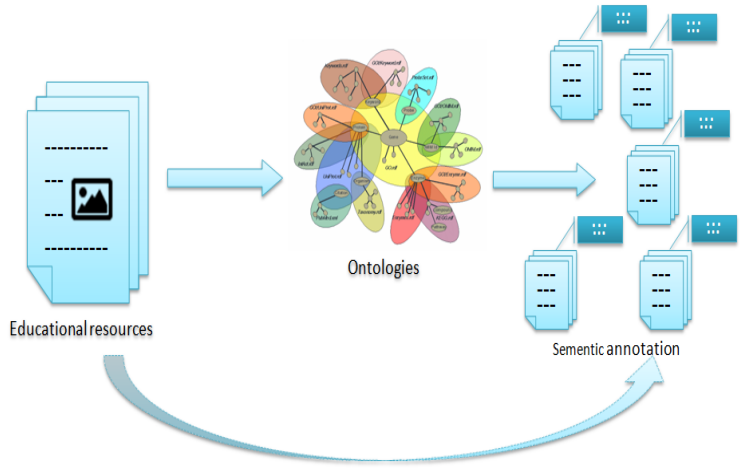

Figure 4. Semantic Annotation Based on Ontologies

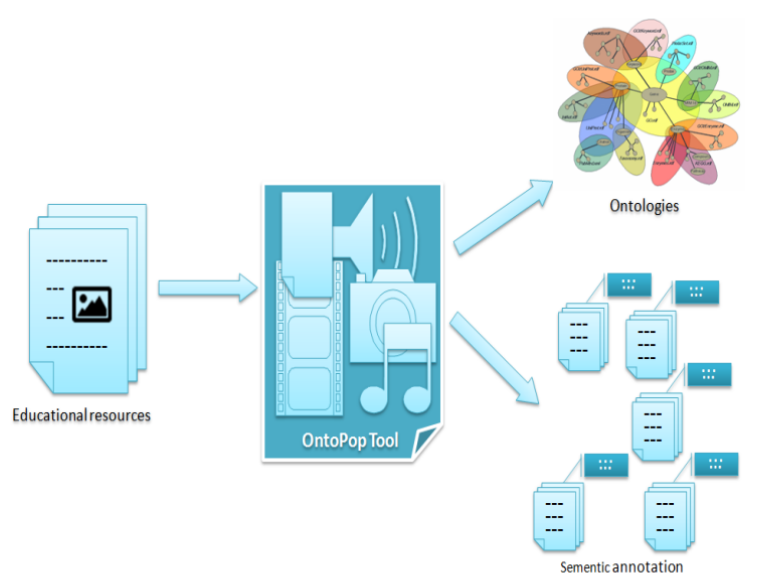

Figure 5. Semantic Annotation Based OntoPop tool

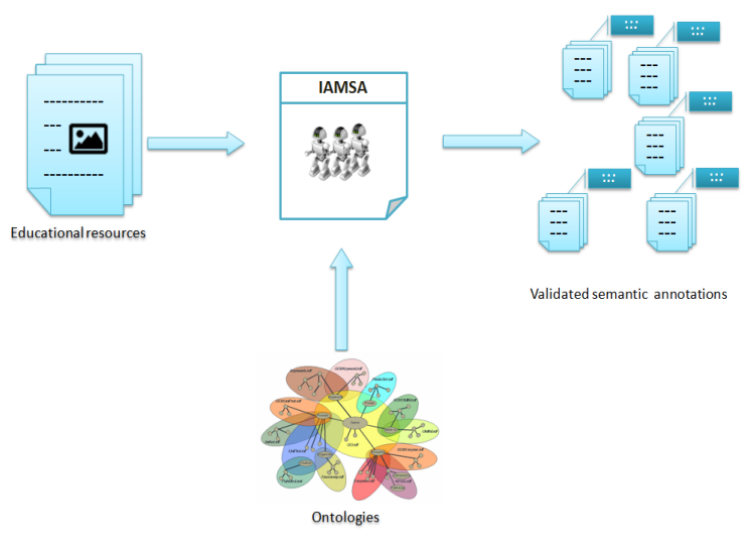

Figure 6. Our Semantic Annotation Solution Based Agents

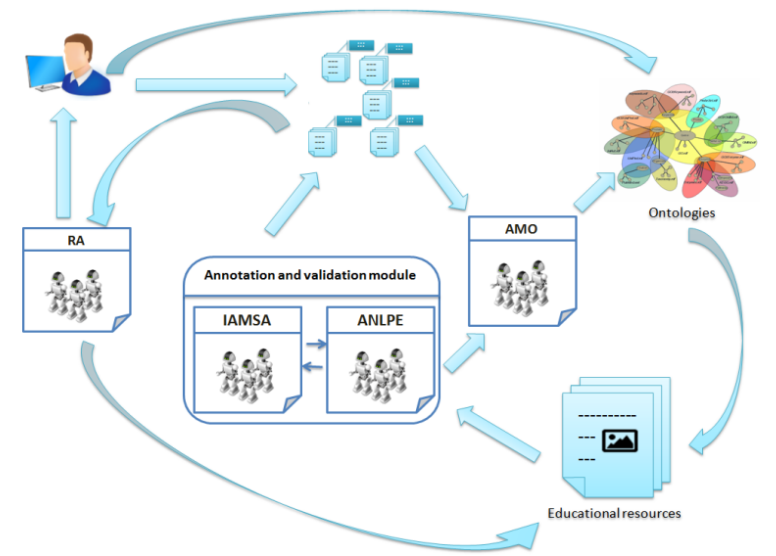

Figure 7. General Pattern of Our solution Annotation of Educational Resources 


\section{REFERENCES}

[1] Abderrahman Chekry, Mohamed Khaldi, « Semantic Web Technologies For The Reuse And Adaptation Of Educational Documents In E-Learning », International Journal of Engineering Pedagogy (iJEP), Volume 3, Issue 1, January 2013 http://dx.doi.org/10.3991/ijep.v3i1.2167

[2] Benayache A. Construction d'une mémoire organisationnelle de formation et évaluation dans un contetxe e-learning le projet Memorae. Thèse de Doctorat, spécialité Informatique, Université de Technologie de Compiègne,2005.

[3] Yannick P., Nack F., Annotations et Intelligence Artificielle, LIRIS FRE 2672 CNRS-Université Claude Bernard Lyon 1, 19/02/07.

[4] Berners-Lee T., Weaving the Web, Harper Eds, San Francisco, 1998, 226 p.

[5] Handschuh S. et Staab S. (Eds).. Annotation for the semantic web. Vol. 96 of Frontiers in Artificial Intelligence and Applications, IOS Press. 2003.

[6] Amardeilh F., Web sémantique et Informatique Linguistique : Propositions méthodologiques et réalisation d'une plate forme logicielle, thèse de doctorat, Université Paris-x-Nanterre, 10 mai 2007.

[7] Nilsson M., Palmer M., Semantic Web Metadata for e-Learning Some Architectural Guidelines. 11th World Wide Web Conference (WWW2002). Hawaii, USA. 2002.

[8] Yannick Prié*, Serge Garlatti. Méta-données et annotations dans le Web sémantique. LIRIS FRE 2672 CNRS - Université Claude Bernard Lyon 1, Laboratoire2 Département IASC GET - ENST Bretagne.

[9] Jean Charlet, Bruno Bachimont, Raphaël Troncy, Ontologies pour le Web sémantique, Mission de recherche STIM, AP-HP \& INSERM ERM 0202, Institut National de l'Audiovisuel, Université Technologique de Compiègne, ISTI-CNR

[10] WOODS W. A. (1991). Understanding subsumption and taxonomy: A framework for progress. In J. F. SOWA,Ed.,Principles of Semantic Networks, chapter 1, p. 45-94. San Mateo, Ca: Morgan Kaufmann Publishers.

\section{AUTHORS}

CHEKRY Abderrahman received the Master degree in software quality in 2009 from Abdelmalek Essaâdi University in Tetwan, Morocco. Currently, he is a $\mathrm{PhD}$ Student in Computer Science. He is also member of Network of Researchers in Information Technology and Communication for Education "Réseau de Chercheurs en Technologies de l'Information et de la Communication pour l'Enseignement" (RES@TICE). Ongoing research interests: Semantic Web and Computing environments for Human Learning "Environnements Informatique pour l'Apprentissage Humain (EIAH)".

ORICHE Aziz received the Master degree in software quality in 2009 from Abdelmalek Essaâdi University in Tetwan, Morocco. Currently, he is a $\mathrm{PhD}$ Student in Computer Science.Ongoing research interests: Semantic Web and Computing environments for Human Learning "Environnements Informatique pour l'Apprentissage Humain (EIAH)".

KHALDI Mohamed received the $\mathrm{Ph}$ degree in Information and Communication Technologies in Education "ICTE". Currently he is a Professor of Computer Engineering in Abdelmalek Essaâdi University and he is the vice-director of the "Ecole Normale Supérieure (ENS)" of Martil in Morocco. He has published several articles on Elearning and Semantic Web. He is also part of many boards of international journals and international conferences.

Submitted 01 June 2013. Published as re-submitted by the authors 23 January 2014. 